\title{
Load Profile-Based Power Loss Estimation for Distribution Networks
}

\author{
Nassim Iqteit (D), Ayşen Basa Arsoy (D), Bekir Çakır \\ Department of Electrical Engineering, Kocaeli University School of Engineering, Kocaeli, Turkey
}

Cite this article as: N. Iqteit, A. Basa Arsoy, B. Çakır, “Load Profile-Based Power Loss Estimation for Distribution Networks", Electrica, vol. 18, no: 2, pp. 275-283, 2018.

\begin{abstract}
In this paper, simple approximate formulas are established to estimate active and reactive power losses in distribution networks. The developed formulas based on load profiles are derived from Elgerd's power loss formulas. A simple model is also proposed to estimate the load profile while considering several parameters, such as the average load demand and the load limitations. Derivation of the load model and the loss formulas are presented along with their applications on two distribution networks. The results show that the loss formulas, which depend on the proposed load profile, can estimate the power losses in distribution networks with high precision.
\end{abstract}

Keywords: load profile model; power losses; distribution network; distribution generator

\section{Corresponding Author: \\ Nassim lqteit}

E-mail:

nassimiqteit@gmail.com

Received: 06.03.2018

Accepted: 10.04 .2018

(c) Copyright 2018 by Electrica

Available online at

http://electrica.istanbul.edu.tr

DOI: 10.26650/electrica.2018.02248

\section{Introduction}

The load profiles in conventional networks can be estimated from the database of the distribution system, while those in smart networks can be obtained directly on time from the smart systems. Estimated load profiles in conventional distribution networks help electrical companies in many fields through such assistance as covering the energy demands of consumers, taking on economic and management decisions, and improving the performance of networks, among others. Many studies were carried out to determine the model of load profiles; the analytical model for determining uncertainty in distribution loads presented in depends on the calculation of mean and variance of the loading profile in each distribution transformer [1]. Daily load profiles for all load types carried out in are modeled depending on the mean, the standard deviation, and the normal distribution of load profiles [2]. The daily load profile in different seasons were also studied in [3]. Previous studies on load profiles needed to find the mean and standard deviation in constructing a load profile model. The proposed load profile model in this paper consists of a small amount of load data; the type of demands and their average values, the minimum and maximum limitations, and the probability of load distribution between the given limitations.

The estimation of active and reactive power loss in distribution systems is one of the main goals for any electric utility company. For example, a reduction of real power loss increases the performance of distribution networks and decreases their financial costs. Additionally, a reduction of reactive power loss reduces decreased voltage and reactive power consumption, while improving the load ability of networks [4]. Subsequently, many equations which depend on generated or consumed power have been proposed to calculate the power loss in transmission and distribution systems, such as: Kron's loss equation, the Branch power loss equation, and Elgerd's loss equation [5-7]. These equations were used in the calculation of power loss in the electrical system, the economic dispatch analysis, and in determining the optimal size and location of distributed generators or capacitors in primary distribution networks [4-6, $8,9-12]$. These equations relate to single values of power (load, generation, or injections pow- 
er), but they do not have load profile functions with time (i.e. daily, monthly or yearly load profiles) in order to calculate the average power losses in electrical networks. This paper, along with the article in [13], presents the full derivation of simple approximation power loss formulas to estimate active and reactive power loss, taking load profiles into account. Additionally, the validity of these formulas is examined in this article by two distribution systems.

This article sheds light on: a simple model of load profiles and power loss formulas based on load demand profiles, modified power flow equations based on power profiles, an algorithm to find active and reactive power loss profiles, and applications on 13 and 69 bus distribution test systems and their simulation results. Finally, the article summarizes the contributions and conclusions of this work.

\section{Simple Approximated Model of Load Profiles}

The profiles of residential, commercial, and industrial load demands were modeled through field measurements and statistical study [2]. The daily load profiles of $P(t)$ or $Q(t)$ at specific probability can be obtained using normal distribution, where the Equation (1) is the general daily load profile.

$$
X_{G}(t)=\mu(t)+\kappa_{1} \sigma(t)
$$

where: $X_{G}(t)$ : general daily load profile, $\mu(t)$ : the mean curve of certain consumer, $\sigma(t)$ : the standard deviation curve of a certain consumer based on daily load curves, and $\mathrm{k}_{1}$ : the Gaussian distribution value at specific probability $\operatorname{Pr}(\%)$. For example, $\mathrm{K}_{1}=1.3$ at $\operatorname{Pr}=90 \%$ and $\mathrm{K}_{1}=1.65, \operatorname{Pr}=95 \%$. The following proposed procedures present a simple and approximated model of daily load profile, and depend upon a small amount of load data:

- $\quad$ According to Equation (1), if the $\sigma(t)=0$, the daily load curve is equal to the mean curve. Under this condition, the daily load profile can be defined as $X_{G}(t)=\mu(t)=\mu_{p u}(t) \times$ average $\{\mu(t)\}$. At zero standard deviation, we can assume $\mu_{p u}(t)$ is the general behavior of residential, commercial, or industrial load profiles in a per unit system where

$X_{\text {base }}=\operatorname{average}\{\mu(t)\}=\frac{\text { monthly consumption }(\mathrm{kWh})}{24 \times 30}$

The per-unit mean residential, commercial, and industrial profiles were obtained through the results of [2], where the area under each curve is equal to one. Figure 1 shows the per unit mean profiles of residential, commercial, and industrial load demands.

- Approximated load profiles can be derived which are based on the values of average and minimum-maximum limits of load demands, as shown below:

$X_{G}(t)=\mu_{p u}(t) X_{\text {base }}+\kappa_{1} \sigma(t)$
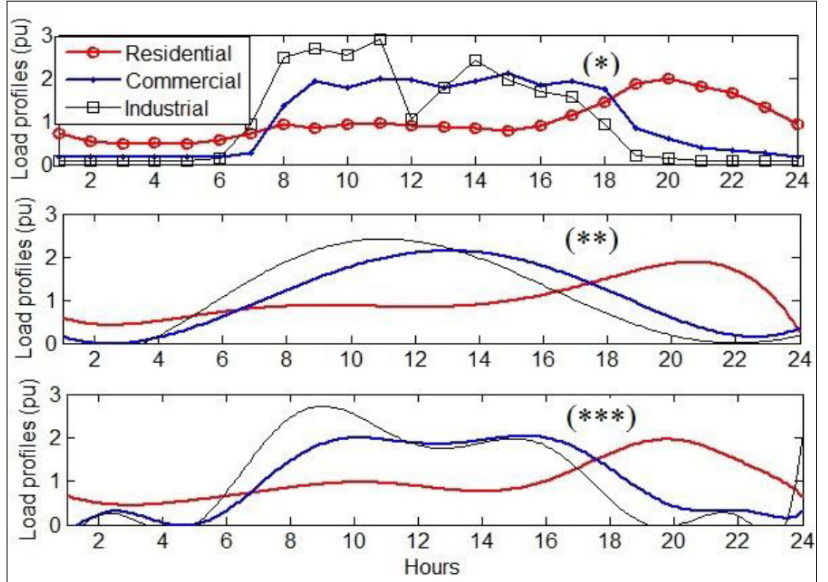

Figure 1. The per unit mean load profiles based on: $\left(^{*}\right)$ the measurement data [2] $\left(^{* *} 5^{\text {th }}\right.$ order polynomial function of curves in $\left(^{*}\right)$ $\left(^{* * *}\right) 10^{\text {th }}$ order polynomial function of curves in $\left(^{*}\right)$

where

$$
\begin{aligned}
& \sigma(t)=\sqrt{E\left(\left[X_{r}(t)\right]^{2}\right)-\mu(t)^{2}} \\
& \sigma(t)=\mu(t) \sqrt{\lambda-1}=\mu_{p u}(t) X_{\text {base }} \sqrt{\lambda-1}
\end{aligned}
$$

where

$\lambda=\frac{\frac{1}{N d} \sum_{i=1}^{N d}\left[x_{i}(t)\right]^{2}}{\left[\frac{1}{N d} \sum_{i=1}^{N d} x_{i}(t)\right]^{2}}$

$E\left(X_{r}\right)=\mu$ is the mean value of $X_{r} . X_{r}$ is the random variable in the set $\left\{x_{1}, x_{2}, \ldots, X_{N d}\right\}$. The subset $\left\{x_{\max }(t), \mu(t), x_{\min }(t)\right\}$ represents the maximum deviation in the set of $X_{r}$. This subset can be used to find out the approximate value of the $\lambda$ ratio. The approximate value of $\lambda$ is shown in Equation (6):

$\lambda \approx \frac{\frac{1}{3}\left[x_{\max }^{2}(t)+\mu^{2}(t)+x_{\min }^{2}(t)\right]}{\left[\frac{1}{3}\left[x_{\max }(t)+\mu(t)+x_{\min }(t)\right]\right]^{2}}$

Figure 2 clarifies the relationship between the maximum, minimum, and mean functions of general load demands and their average values. Replacing the average values of these functions instead of those of the general load functions is the second approximation that can be used to simplify the calculation of the $\lambda$ ratio where the symbol is the average of $x(t)$. Equation (7) represents the approximate value of $\lambda$, based on the range of load limits:

$\lambda \approx \frac{\frac{1}{3}\left[\bar{x}_{\text {max }-p u}^{2}+\bar{\mu}_{p u}^{2}+\bar{x}_{\text {min-pu }}^{2}\right]}{\left[\frac{1}{3}\left[\bar{x}_{\text {max }-p u}+\mu_{p u}+\bar{x}_{\text {min-pu }}\right]\right]^{2}}=\frac{3\left[\bar{x}_{\text {max }-p u}^{2}+\bar{x}_{\min -p u}^{2}+1\right]}{\left[\left[\bar{x}_{\text {max }-p u}+\bar{x}_{\min -p u}+1\right]\right]^{2}}$

Now Equation (1) can be reformulated based on the approximate value of $\lambda$ as shown in Equation (8). Table 1 displays three numerical examples and the comparison between exact and approximated values of the $\lambda$ ratio, and profile values of $X_{G}$. 
Table 1. Examples compare between exact and approximate values of load profiles at specific times.

\begin{tabular}{llllll}
\hline & & \multicolumn{2}{c}{$\lambda$ ratio } & \multicolumn{2}{c}{ The value of profile XG } \\
\cline { 2 - 5 } Profile of set $X_{r}$ at time $\mathbf{~}$ & Subset of $\mathbf{X}^{r}$ & Exact & Appx & Exact & Appx \\
\hline$\{0.9,1.075,1.1,0.95,0.9,1,0.97,0.94,1.085,0.96\}$ & $\{1.1,0.9,0.988\}$ Range $\approx \pm 10 \%$ & 1.00512 & 1.00675 & 1.080 & 1.094 \\
\hline$\{0.8,1.25,0.75,0.7,1.3,1,1.1,1.2,1.15,0.85\}$ & $\{1.3,0.7,1.01\}$ Range $\approx \pm 30 \%$ & 1.04304 & 1.05962 & 1.282 & 1.331 \\
\hline$\{1.4,1.3,0.55,0.7,1.49,1,0.51,0.86,1.39,0.86\}$ & $\{1.49,0.51,1.006\}$ Range $\approx \pm 50 \%$ & 1.11992 & 1.15944 & 1.459 & 1.528 \\
\hline
\end{tabular}

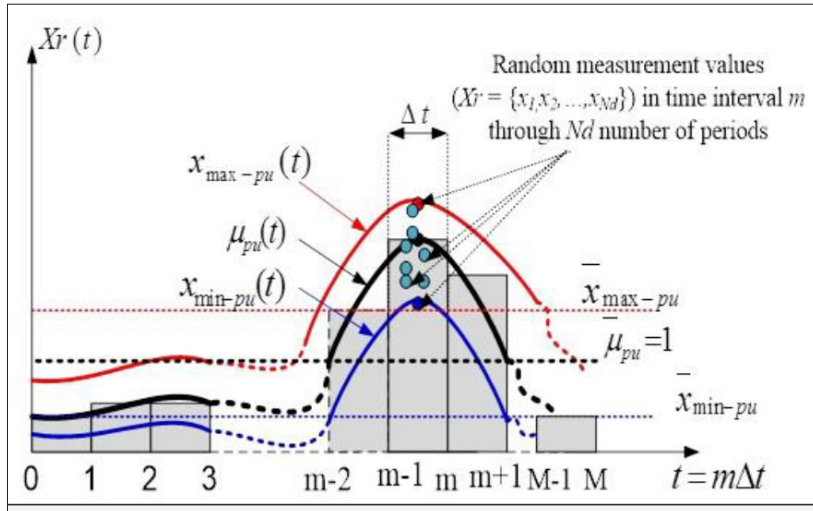

Figure 2. Maximum, minimum, and mean functions of the general load demand based on random subset of $\operatorname{Xr}(m)$

$X_{G}(t)=\mu_{p u}(t) X_{\text {base }}\left(1+\kappa_{1} \sqrt{\lambda-1}\right)$

\section{Power Loss}

\section{Exact Power Loss Formulas}

The total active and reactive power lost in a distribution network with $N$ buses can be calculated by Elgerd's Equations (9) and (10), respectively [4]. The equations show the power loss depending on the active and reactive power injections,

$$
\begin{aligned}
& P_{L}=\mathbf{P}^{\prime} \boldsymbol{\alpha} \mathbf{P}+\mathbf{Q}^{\prime} \boldsymbol{\alpha} \mathbf{Q}+\mathbf{Q}^{\prime} \boldsymbol{\beta} \mathbf{P}-\mathbf{P}^{\prime} \boldsymbol{\beta} \mathbf{Q} \\
& Q_{L}=\mathbf{P}^{\prime} \boldsymbol{\gamma} \mathbf{P}+\mathbf{Q}^{\prime} \gamma \mathbf{Q}+\mathbf{Q}^{\prime} \xi \mathbf{P}-\mathbf{P}^{\prime} \xi \mathbf{Q}
\end{aligned}
$$

where

$$
\mathbf{P}^{\prime}=\left[\begin{array}{llllll}
P_{1} & P_{2} & \ldots & P_{i} & \ldots & P_{N}
\end{array}\right], \mathbf{Q}^{\prime}=\left[\begin{array}{llllll}
Q_{1} & Q_{2} & \ldots & Q_{i} & \ldots & Q_{N}
\end{array}\right],
$$
and

$a=\left[a_{i j}\right], \beta=\left[\beta_{i j}\right], \gamma=\left[\gamma_{i j}\right]$ and $\xi=\left[\xi_{i j}\right]$. While the size of matrices $a$, $\beta, \gamma$ and $\xi$ is $N \times N$, the coefficients $a_{i j}, \beta_{i j} \gamma_{i j}$ and $\xi_{i j}$ are functions of the magnitude and angle of phasor voltage. Also, $a_{i j}$ and $\beta_{i j}$ depend on the resistive entries $r_{i j}$ of the impedance matrix $\left[Z_{i j}\right]$, while $\gamma_{i j}$ and $\xi_{i j}$ depend on the reactance entries $x_{i j} P_{i}$ and $P_{j}$ are the active power injections of buses $i$ and $j$, respectively; $Q_{i}$ and $Q_{j}$ are the reactive power injections of buses $i$ and $j$, respectively, where the power injection is the difference between the power generations and load demands for the same bus.

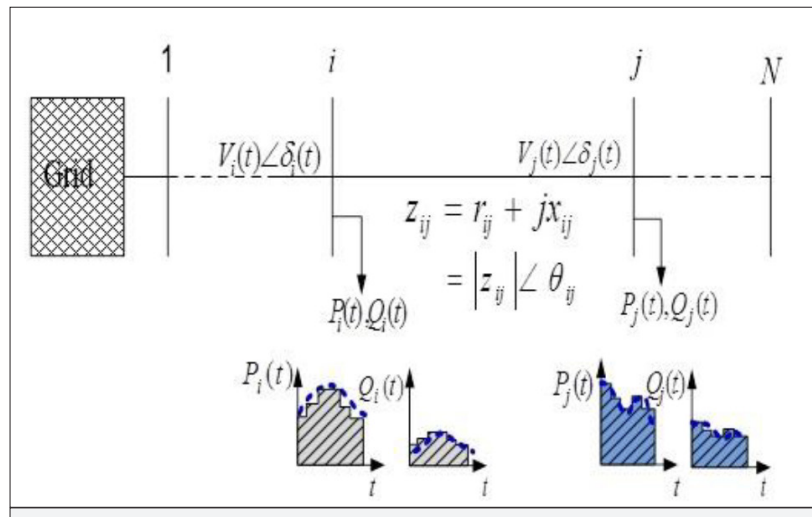

Figure 3. Load profiles on a distribution network

\section{Power Loss Formulas Based on Load Profiles}

Figure 3 displays the change of the power injections on the nodes of a distribution network at each moment of time. The distribution system was assumed perfectly balanced in the three-phase system. The load profiles and the line segments were supposed to be perfectly balanced as well. The line impedance of each segment was consistent with the time, but the state variables $\left(V_{i}(t)\right.$ and $\left.\delta_{i}(t)\right)$ were not consistent because they depend on the power demands.

\section{Linearization Principle and Average Integral of Power Profiles}

A linearization technique and an additive property of integrals were used to derive a simple power loss equation based on power profiles. Profiles of active and reactive load demands, power injections, and power loss are nonlinear functions with time variables. Additionally, active and reactive power loss relations are nonlinear with profiles of active and reactive power injections. Figure 4 shows a general nonlinear function $X(t)$, where $X(t)$ could represent the power profiles of load demands $\left(P_{D i}(t), Q_{D i}(t)\right)$, power generations $\left(P_{G i}(t), Q_{G i}(t)\right)$, power injections $\left(P_{i}(t), Q_{i}(t)\right)$ at node $i$, or power loss $\left(P_{L T}(t), Q_{L T}(t)\right)$.

Through one period $T$ the function $X(t)$ is divided by $M$ regular intervals with a length of $\Delta t$ for each one. The length of the regular interval ( $\Delta t=\frac{T}{M}$ ) depends on the power measurement devices (smart, digital, or conventional meters) or the accura- 


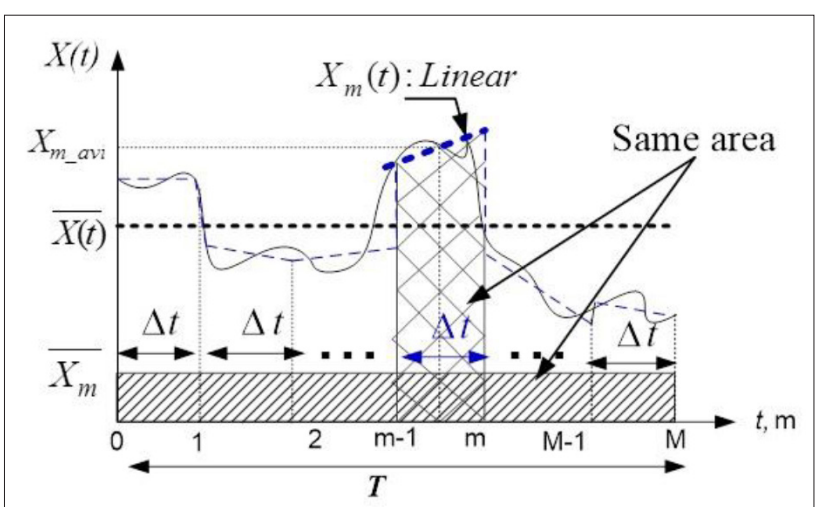

Figure 4. Linearization principle of the general nonlinear function $X(t)$

cy of analysis. $X(t)$ was considered approximate linear function through each interval $m$, where $X_{m}(t)$ is the linear approximation of $X(t)$ over interval $m . X_{m \text { avi }}$ is the average integral value of $X_{m}(t)$ through time $\Delta t . \bar{X}_{m}$ is the weight of the average integral value of $X(t)$ in interval $m$.

The following relations were obtained by using Figure 4:

- The length of the regular interval $m$ is $\Delta t=\frac{T}{M}$ and the endings of this interval are $(m-1) \frac{T}{M}$ and $\frac{T}{M}$, where $m=1,2, \ldots, M$. If $X(t)$ is assumed a linear function along interval $m$, then the values of $X_{m \_a v i}$ and $\bar{X}_{m}$ can be approximated by Equation (11).

$\left\{\begin{array}{c}X_{m_{-} a v i}=\frac{1}{\Delta t_{m}} \int_{(m-1) \frac{T}{M}}^{m \frac{T}{M}} X_{m}(t) d t \cong X\left(\left(m-\frac{1}{2}\right) \frac{T}{M}\right) \\ \bar{X}_{m}=\frac{1}{T} \int_{(m-1) \frac{T}{M}}^{m \frac{T}{M}} X(t) d t \cong \frac{1}{M} X\left(\left(m-\frac{1}{2}\right) \frac{T}{M}\right)\end{array}\right\}$

The average integral of $X(t)$ through the period $T$ is approximated in Equation (12)

$\overline{X(t)}=\frac{1}{T} \int_{0}^{T} X(t) d t=\sum_{m=1}^{M} \frac{1}{T} \int_{(m-1) \frac{T}{M}}^{m \frac{T}{M}} X(t) d t=$

$\sum_{m=1}^{M} \bar{X}_{m} \Rightarrow$

$\overline{X(t)} \cong \frac{1}{M} \sum_{m=1}^{M} X_{m} \cong \frac{1}{M} \sum_{m=1}^{M} X\left(\left(m-\frac{1}{2}\right) \frac{T}{M}\right)$

\section{Approximated Power Loss Formulas Based on Load Profiles}

According to Equation (11), over interval $m$ the average integral of the power injection profile $\left(P_{i}(t), Q_{i}(t), P_{j}(t)\right.$ or $\left.Q_{j}(t)\right)$ can be represented by single point and constant value at time $t_{b}=\left(m-\frac{1}{2}\right) \frac{T}{M}$. Dependent on approximated values of power injection profiles, the active power loss profile for each interval $m$ was derived in the following equation:

$$
\begin{aligned}
& P_{L T m}=P_{L T}\left(t_{b}\right)=f\left(P_{i}\left(t_{b}\right), P_{j}\left(t_{b}\right), Q_{i}\left(t_{b}\right), Q_{j}\left(t_{b}\right)\right) \\
& =f\left(P_{m i}, P_{m j}, Q_{m i}, Q_{m j}\right) \\
& P_{L T m}=\boldsymbol{P}_{\boldsymbol{m}}^{\prime} \boldsymbol{\alpha}_{m} \boldsymbol{P}_{m}+\boldsymbol{Q}_{\boldsymbol{m}}^{\prime} \boldsymbol{\alpha}_{m} \boldsymbol{Q}_{m} \\
& +\boldsymbol{Q}_{\boldsymbol{m}}^{\prime} \boldsymbol{\beta}_{\boldsymbol{m}} \boldsymbol{P}_{\boldsymbol{m}}-\boldsymbol{P}_{\boldsymbol{m}}^{\prime} \boldsymbol{\beta}_{\boldsymbol{m}} \boldsymbol{Q}_{\boldsymbol{m}}
\end{aligned}
$$

Similarly, the reactive power profile for each interval $m$ can be calculated using Equation (13) only by replacing $\gamma_{m}$ and $\xi_{m}$ instead of $a_{m}$ and $\beta_{m^{\prime}}$, respectively.

According to Equations (12) and (13), the average approximation of active and reactive power loss based on load profiles is obtained using Equations (14) and (15), respectively

$$
\begin{aligned}
& P_{L T}=\frac{1}{M} \sum_{m=1}^{M}\left[\begin{array}{c}
\mathbf{P}_{\boldsymbol{m}}^{\prime} \boldsymbol{\alpha}_{m} \mathbf{P}_{m}+\mathbf{Q}_{m}^{\prime} \boldsymbol{\alpha}_{m} \mathbf{Q}_{m} \\
+\mathbf{Q}_{\boldsymbol{m}}^{\prime} \boldsymbol{\beta}_{m} \mathbf{P}_{m}-\mathbf{P}_{\boldsymbol{m}}^{\prime} \boldsymbol{\beta}_{m} \mathbf{Q}_{m}
\end{array}\right] \\
& Q_{L T}=\frac{1}{M} \sum_{m=1}^{M}\left[\begin{array}{c}
\mathbf{P}_{\boldsymbol{m}}^{\prime} \boldsymbol{\gamma}_{m} \mathbf{P}_{m}+\mathbf{Q}_{\boldsymbol{m}}^{\prime} \boldsymbol{\gamma}_{m} \mathbf{Q}_{m} \\
+\mathbf{Q}_{\boldsymbol{m}}^{\prime} \boldsymbol{\xi}_{m} \mathbf{P}_{m}-\mathbf{P}_{\boldsymbol{m}}^{\prime} \boldsymbol{\xi}_{m} \mathbf{Q}_{m}
\end{array}\right]
\end{aligned}
$$

$$
\begin{aligned}
& \text { where } \\
& \mathbf{P}_{\boldsymbol{m}}^{\prime}=\mathbf{P}^{\prime}\left(\left(m-\frac{1}{2}\right) \frac{T}{M}\right)=\left[\begin{array}{lllll}
P_{m 1} & P_{m 2} & \ldots & P_{m i} \ldots P_{m N}
\end{array}\right]
\end{aligned}
$$

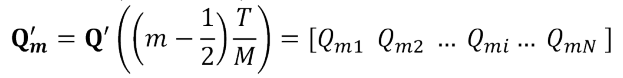

$$
\begin{aligned}
& P_{m i}=P_{i}\left(\left(m-\frac{1}{2}\right) \frac{T}{M}\right) \text { and } Q_{m i}=Q_{i}\left(\left(m-\frac{1}{2}\right) \frac{T}{M}\right) \\
& \boldsymbol{\alpha}_{m}=\boldsymbol{\alpha}\left(\left(m-\frac{1}{2}\right) \frac{T}{M}\right)=\left[\alpha_{m i j}\right], \quad \boldsymbol{\beta}_{\boldsymbol{m}}=\boldsymbol{\beta}\left(\left(m-\frac{1}{2}\right) \frac{T}{M}\right)= \\
& {\left[\beta_{m i j}\right], \quad \gamma_{m}=\gamma\left(\left(m-\frac{1}{2}\right) \frac{T}{M}\right)=\left[\gamma_{m i j}\right], \xi_{m}=\xi\left(\left(m-\frac{1}{2}\right) \frac{T}{M}\right)=\left[\xi_{m i j}\right]}
\end{aligned}
$$

where the size of matrices $a_{m^{\prime}} \beta_{m^{\prime}} \gamma_{m}$ and $\xi_{m}$ is $N \times N$. The coefficients $a_{m i j}, \beta_{m i j}, \gamma_{m i j}$ and $\xi_{m i j}$ are calculated for each interval $m$,

where

$$
\begin{aligned}
& \alpha_{m i j}=\alpha_{i j}\left(\left(m-\frac{1}{2}\right) \frac{T}{M}\right)=\frac{r_{m i j}}{V_{m i} V_{m j}} \cos \left(\delta_{m i}-\delta_{m j}\right) \\
& \beta_{m i j}=\beta_{i j}\left(\left(m-\frac{1}{2}\right) \frac{T}{M}\right)=\frac{r_{m i j}}{V_{m i} V_{m j}} \sin \left(\delta_{m i}-\delta_{m j}\right) \\
& \gamma_{m i j}=\gamma_{i j}\left(\left(m-\frac{1}{2}\right) \frac{T}{M}\right)=\frac{x_{m i j}}{V_{m i} V_{m j}} \cos \left(\delta_{m i}-\delta_{m j}\right) \\
& \xi_{m i j}=\xi_{i j}\left(\left(m-\frac{1}{2}\right) \frac{T}{M}\right)=\frac{x_{m i j}}{V_{m i} V_{m j}} \sin \left(\delta_{m i}-\delta_{m j}\right)
\end{aligned}
$$

where

$r_{m i j}=r_{i j}, x_{m i j}=x_{i j}, \alpha_{m i j}=\alpha_{m j i}, \beta_{m i j}=-\beta_{m j i}$, $\gamma_{m i j}=\gamma_{m j i}, \xi_{m i j}=-\xi_{m j i}$.

\section{Algorithm of Power Loss Estimation}

The coefficients $a_{m i j^{\prime}} \beta_{m i j^{\prime}} \gamma_{m i j}$, and $\xi_{m i j}$ are calculated for each interval $m$. Power flow equations in reference [5] were revised to include the state variables and power injections at each value of $m$, where the state variables $V_{m i}$ and $\delta_{m i}$ can be calculated by solving the modified power flow equations (17) and (18). Numerical methods such as the Newton-Raphson method are used to solve equations (17) and (18). The power injections $P_{m i}$ and $Q_{m i}$ are calculated by Equation (11).

$P_{m i}=\sum_{j=1}^{N} V_{m i} V_{m j} Y_{m i j} \cos \left(\theta_{m i j}-\delta_{m i}+\delta_{m j}\right)(17)$ 


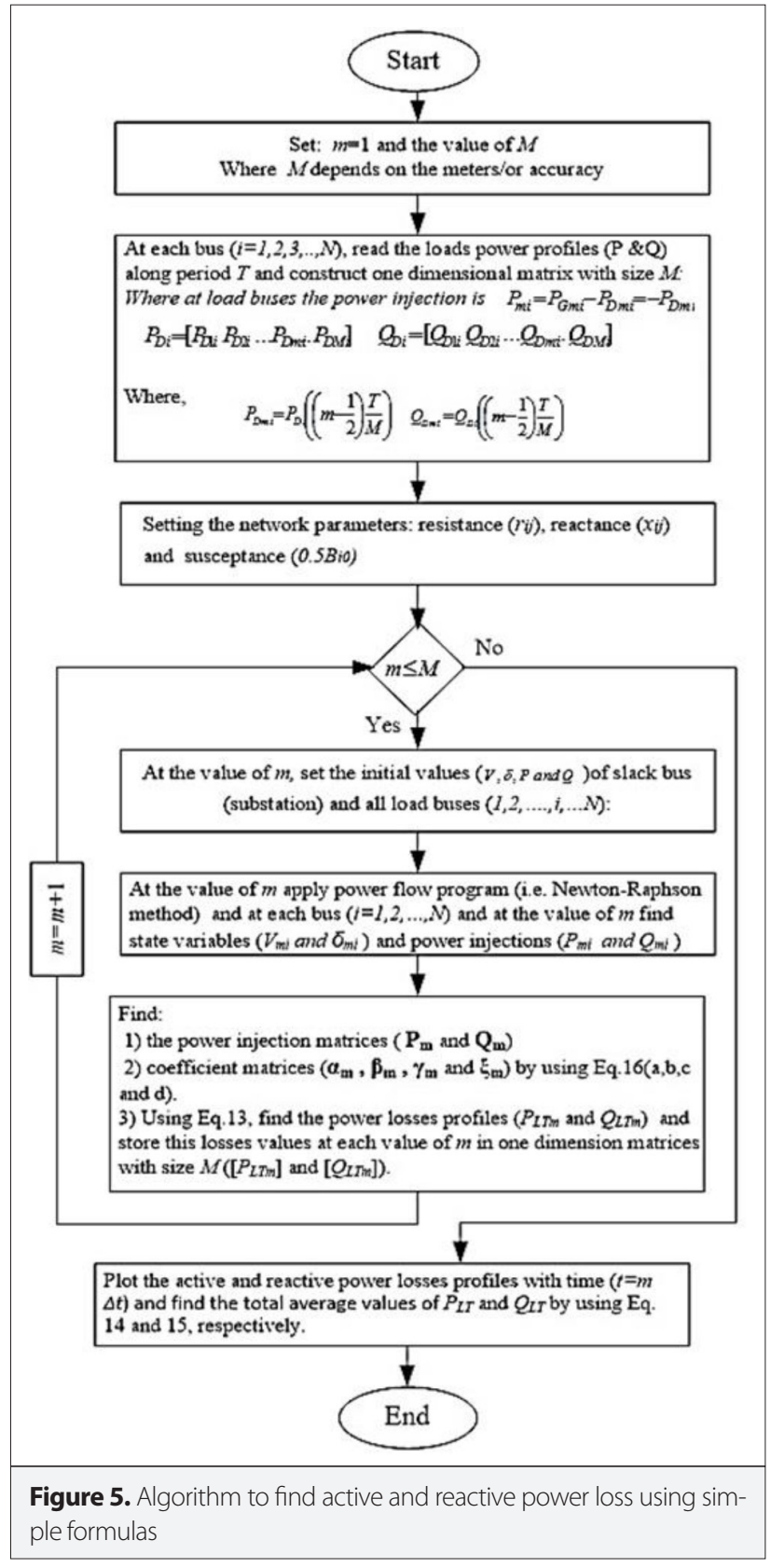

$Q_{m i}=-\sum_{j=1}^{N} V_{m i} V_{m j} Y_{m i j} \sin \left(\theta_{m i j}-\delta_{m i}+\delta_{m j}\right)$

where $Y_{m i j}$ is the entry of admittance matrix at values of $m, i$, and $j$. Figure 5 represents an algorithm to find the profiles and the total average of active and reactive power loss.

\section{Applied Examples}

\section{The 13 Bus System}

A test system in [13] having 18MVA and $12.5 \mathrm{kV}$ specifications is shown in Figure 6. This system load data is given in Table 2. The base power of this network is $10 \mathrm{MVA}$ at $12.5 \mathrm{kV}$.
Table 2. Load data of a 13 bus radial distribution network

\begin{tabular}{lccccc}
\hline BUS & $\mathbf{P}(\mathbf{p u})$ & $\mathbf{Q}(\mathbf{p u})$ & $\begin{array}{c}\text { Range } \\
\pm \mathbf{R} \%\end{array}$ & $\begin{array}{c}\text { Probability } \\
(\mathbf{P r} \%)\end{array}$ & Type of load \\
\hline 1 & 0 & 0 & - & - & - \\
\hline 2 & 0 & 0 & - & - & - \\
\hline 3 & 0.3045 & 0.1828 & 5 & 90 & $R$ \\
\hline 4 & 0.4059 & 0.2435 & 30 & 90 & $R$ \\
\hline 5 & 3.0351 & 2.2519 & 15 & 90 & $80 \% R+20 \% \mid$ \\
\hline 6 & 0.7060 & 0.4353 & 10 & 90 & $C$ \\
\hline 7 & 1.0509 & 0.7006 & 20 & 90 & I \\
\hline 8 & 1.0631 & 0.6571 & 10 & 90 & $R$ \\
\hline 9 & 0.4578 & 0.2861 & 13 & 90 & $C$ \\
\hline 10 & 0.8299 & 0.5532 & 15 & 90 & $R$ \\
\hline 11 & 0.4450 & 0.2966 & 50 & 90 & $75 \% R+25 \% C$ \\
\hline 12 & 1.0580 & 0.6172 & 20 & 90 & $R$ \\
\hline 13 & 0.3046 & 0.1521 & 5 & 90 & $R$ \\
\hline$R: R 50$ & & & $R$ \\
\hline
\end{tabular}

R: Residential C : Commercial I: Industrial

\section{The 69 Bus System}

The 69 bus distribution system (IEEE test system) displayed in Figure 7 has a total active load of $3.8021 \mathrm{MW}$ and a total reactive load of 2.6945 MVAr. The system data information is given in reference [14]. The base power of this system is 100MVA at $12.66 \mathrm{kV}$. The proposed loss formulas and estimated load model were tested on this system while two wind DGs and one photovoltaic DG were used. The location and ratings of these DGs are given in Table 3. Also, the generation profiles of the DGs are given in reference [9].

\section{Simulation Results and Discussion}

The proposed load profile model has been used to estimate the load profiles at each node of the 13 and 69 bus systems. The type of load, the minimum and maximum limitations, and the probability of load distribution as tabulated in Table 2 were considered, along with the mean load value to estimate each load profile at each node of the 13 bus system. The load profiles and DGs of the 69 bus system were estimated by using the type of loads and DGs with their mean values. It's clear that the factor $\lambda$ was assumed equal to one in this system.

\section{Simulation Results of the 13 Bus System}

Figure 8 shows the estimated active and reactive load profiles of the 13 bus system at each node of the network. Table 4 shows the average values of load profiles displayed in Figure 8. The 


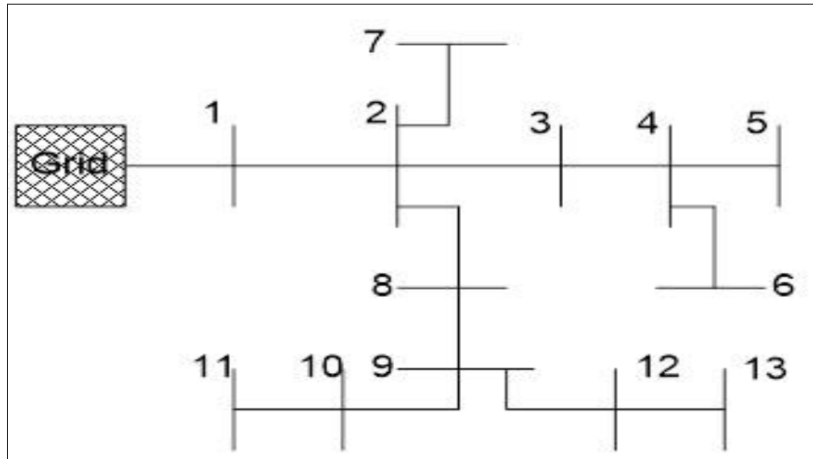

Figure 6. The 13 bus radial distribution network

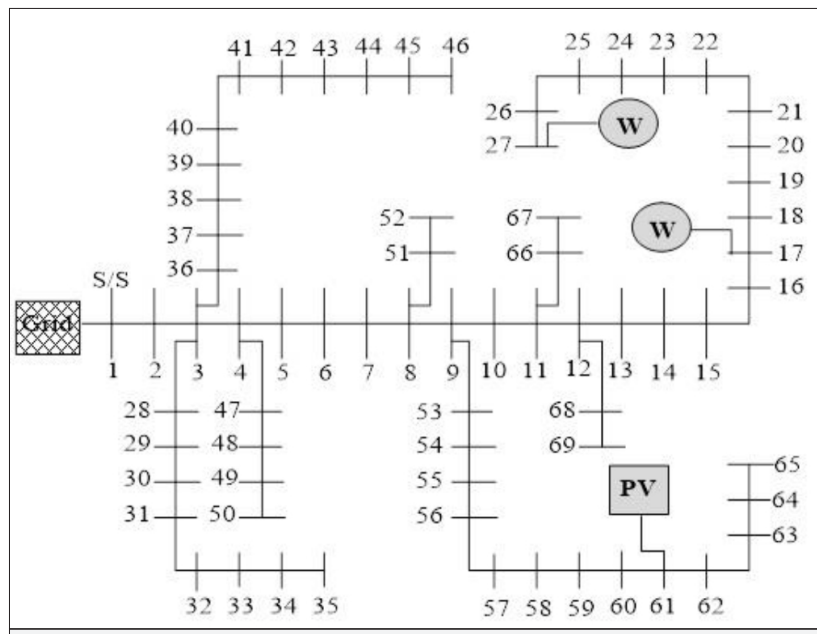

Figure 7. The 69 bus radial distribution network

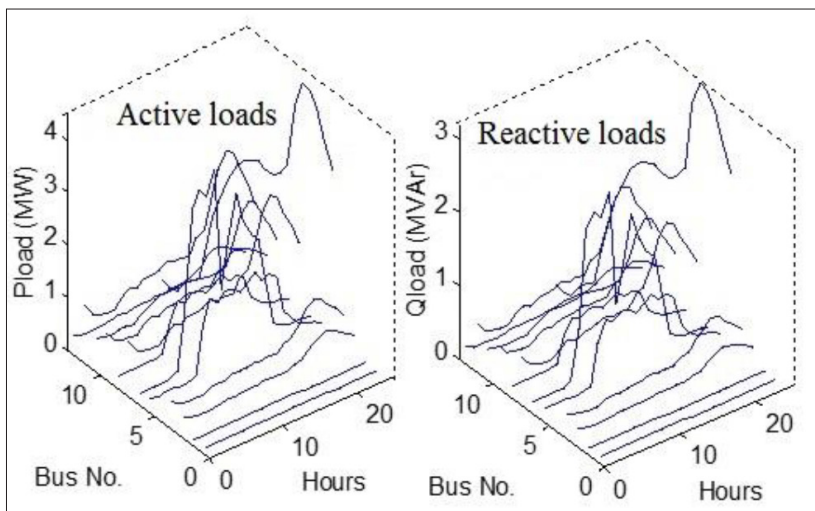

Figure 8. Active and Reactive load profiles of a 13 bus radial distribution network

effects of limitations on load profiles can be noted by comparing the values of $P$ and $Q$ in Tables 2, 4. The estimated load profiles increase by an increasing mean of load demand, a range of load limitations, and a probability factor of $\mathrm{K}_{1}$.

The algorithm for power loss calculations is achieved using MATLAB. Table 5 includes the results of the average power loss of the 13 node radial distribution network. The results were

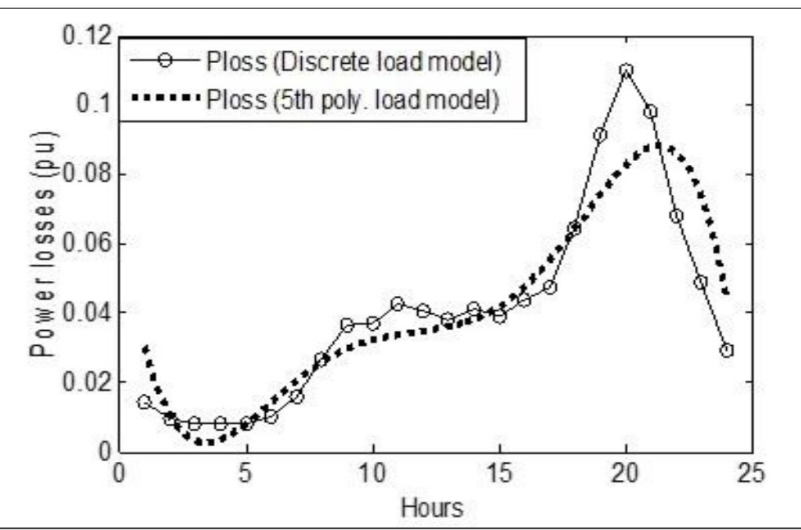

Figure 9. Comparison between the power loss profiles of the 13 bus system using the discrete load model versus the $5^{\text {th }}$ order polynomial load model

Table 3. The rating and location of DGs in the 69 bus system

\begin{tabular}{lccc}
\hline DG type & Wind & wind & PV \\
\hline Location (bus No.) & 17 & 27 & 61 \\
\hline Size (MVA) & 0.559 & 0.613 & 1.7 \\
\hline Power factor & 0.894 & 0.898 & 0 \\
\hline
\end{tabular}

Table 4. Average values of the proposed load profile model

\begin{tabular}{lcc}
\hline bus & $\mathbf{P}_{\text {proposed model }}(\mathbf{p u})$ & $\mathbf{Q}_{\text {proposed model }}(\mathbf{p u})$ \\
\hline 1 & 0 & 0 \\
\hline 2 & 0 & 0 \\
\hline 3 & 0.3207 & 0.1925 \\
\hline 4 & 0.5346 & 0.3207 \\
\hline 6 & 3.5194 & 2.6112 \\
\hline 7 & 0.7815 & 0.4818 \\
\hline 9 & 1.2750 & 0.8500 \\
\hline 10 & 1.1761 & 0.7270 \\
\hline 11 & 0.5210 & 0.3256 \\
\hline 12 & 0.9623 & 0.6415 \\
\hline 13 & 0.6812 & 0.4541 \\
\hline & 1.2830 & 0.7484 \\
\hline 5 & 0.3207 & 0.1601
\end{tabular}

estimated using the load profile and their average and maximum values. Table 5 confirms that the results of the power loss equations are very close to the results of the power lost when it is directly calculated by power flow programs. The power loss values are different when using single values' average/maximum demand and power profiles because the power loss of 
Table 5. Power losses in the 13 bus radial distribution network

\begin{tabular}{lccc}
\hline $\begin{array}{l}\text { Calculations } \\
\text { based on }\end{array}$ & $\begin{array}{c}\text { Active/ } \\
\text { Reactive }\end{array}$ & $\begin{array}{c}\text { losses } \\
\text { formula }\end{array}$ & $\begin{array}{c}\text { Power flow } \\
\text { calculation }\end{array}$ \\
\hline $\begin{array}{l}\text { Maximum load } \\
\text { demands }\end{array}$ & $\mathrm{P}(\mathrm{pu})$ & 0.14660 & 0.14660 \\
\cline { 2 - 4 } $\begin{array}{l}\text { Average load } \\
\text { demands }\end{array}$ & $\mathrm{Q}(\mathrm{pu})$ & 0.26761 & 0.26762 \\
\cline { 2 - 4 } & $\mathrm{Q}(\mathrm{pu})$ & 0.03452 & 0.03453 \\
\hline $\begin{array}{l}\text { Load power } \\
\text { profiles }\end{array}$ & $\mathrm{P}(\mathrm{pu})$ & 0.04070 & 0.065070 \\
\cline { 2 - 4 } & $\mathrm{Q}(\mathrm{pu})$ & 0.07622 & 0.07615 \\
\hline
\end{tabular}

Table 6. Power losses in a 69 bus radial distribution network with two wind DGs and one PV DG

\begin{tabular}{lccc}
\hline $\begin{array}{l}\text { Calculations } \\
\text { based on }\end{array}$ & $\begin{array}{c}\text { Active/ } \\
\text { Reactive }\end{array}$ & $\begin{array}{c}\text { loss } \\
\text { formula }\end{array}$ & $\begin{array}{c}\text { Power flow } \\
\text { calculation }\end{array}$ \\
\hline $\begin{array}{l}\text { Maximum } \\
\text { load demands }\end{array}$ & $\mathrm{P}(\mathrm{pu})$ & 0.004111 & 0.004111 \\
\cline { 2 - 4 } $\begin{array}{l}\text { Average load } \\
\text { demands }\end{array}$ & $\mathrm{P}(\mathrm{pu})$ & 0.0001905 & 0.001905 \\
\hline & $\mathrm{Q}(\mathrm{pu})$ & 0.000343 & 0.000344 \\
\hline Load power profiles & $\mathrm{P}(\mathrm{pu})$ & 0.001566 & 0.001566 \\
\cline { 2 - 4 } & $\mathrm{Q}(\mathrm{pu})$ & 0.000719 & 0.000718 \\
\hline
\end{tabular}

The losses of the system without DG based on load profiles are:

$P_{\text {loss }}=0.004463 p u Q_{\text {loss }}=0.002009 \mathrm{pu}$

the network is a nonlinear function of load profiles. For example, the active power loss based on the load profiles is equal to $0.0407 \mathrm{pu}$, but when based on the average values of these load profiles it is equal to $0.03453 \mathrm{pu}$. The active power loss based on maximum values of the load profiles is equal to $0.1466 \mathrm{pu}$. The calculation of active power loss using Equation (14) is more accurate and effective than using Equation (9); likewise, the method of calculating reactive power loss using Equation (15) is more accurate and effective than using Equation (10).

According to the computed loss profiles, the maximum active and reactive power losses are $0.11 \mathrm{pu}$ and $0.19 \mathrm{pu}$, respectively at 20:00. Figure 9 shows the real power loss profiles based on two types of load models: 1) a discrete load model as shown in Figure $1\left(^{*}\right)$ and 2 ) the $5^{\text {th }}$ order polynomials load model as shown in Figure $1\left(^{* *}\right)$. The average active power loss in discrete mode is $0.04070 \mathrm{pu}$, whereas in the polynomial model it is $0.040707 \mathrm{pu}$. These are almost the same.

\section{Simulation Results of the 69 Bus System}

The estimated active and reactive load profiles of the 69 bus system at each node of the network are illustrated in Figure 10.

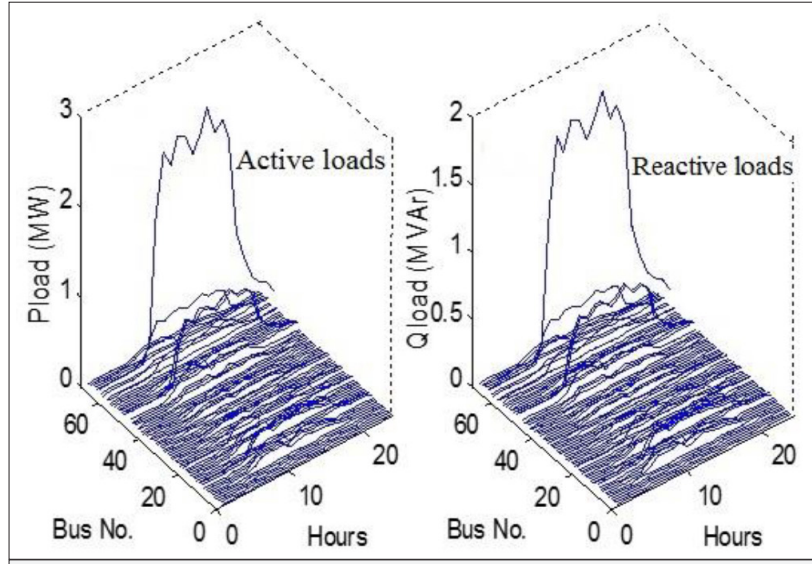

Figure 10. Active and Reactive load profiles of a 69 bus radial distribution network

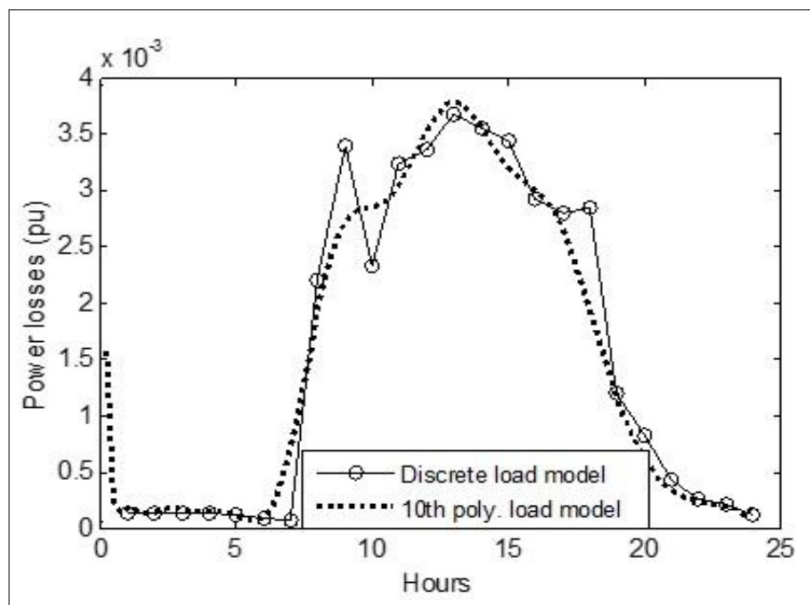

Figure 11. Comparison between the power loss profiles of the 69 bus system using the discrete load model versus the $10^{\text {th }}$ order polynomial load model

The estimated load profiles increase by increasing their mean values. This figure also clarifies that the 69 bus system load is an industrial network.

The active and reactive power losses of the 69 bus system using average/maximum demands, and using load profiles as shown in Figure 10, are represented in Table 6. Additionally, the estimated losses based on the load profiles using Equations (14) and (15) are more precise than the load profiles using Equations (9) and (10), respectively. As can be seen by Table 6, the total system loss after integrating DGs into the system, is lower than the total loss of the system without DG.

According to the calculated loss profiles, the maximum active power loss is $0.003671 \mathrm{pu}$ at $13: 00$. Whereas the maximum reactive loss is 0.001689pu at 9:00. Figure 11 illustrates the real power loss profiles based on the discrete load model and the $10^{\text {th }}$ order polynomials load model as shown in Figure $1\left(^{* * *}\right)$. 
The average active power losses computed for both models are $0.001566 \mathrm{pu}$ and $0.001540 \mathrm{pu}$, respectively.

\section{Conclusion}

This paper presents approximate formulas to estimate the active and reactive power loss in distribution networks based on load demand profiles obtained by a simple approximated model. The load profile model and power loss formulas are examined through the 13 and the 69 bus distribution networks. Types, mean, deviation, and the normal distribution factor of load are the main variables that impact the load profile. The developed loss formulas give more precise results when compared to the power loss formula based on the average/ maximum load demand. The presented formulas of active and reactive power loss offer the following benefits: Easy to apply, capable of using discrete load profiles, and a simulation of results generating only a small error when using an appropriate number of time intervals. The results show that when DGs are integrated into the distribution network the power loss is diminished.

The advantage of the simple approximated formulas can be used not only in power loss analysis but also in economic dispatch analysis and to estimate the optimal setting of distributed generators that can be inserted into distribution networks.

Peer-review: Externally peer-reviewed.

Conflict of Interest: The Authors have no conflicts of interest to declare.

Financial Disclosure: This study was supported by the TUBITAK under project number 114 S47.

\section{References}

1. D. T. Nguyen, "Modeling Load Uncertainty in Distribution Network Monitoring", IEEE Trans Power Syst, vol. 30, no. 5, pp. 2321-2328, 2015.
2. J. A. Jardini, C. M. V. Tahan, M. R. Gouvea, S. U. Ahn, F. M. Figueiredo, "Daily load profiles for residential, commercial and industrial low voltage consumers", IEEE Trans Power Delivery, vol. 15, no. 1, pp. 375-380, 2000.

3. E. C. Bobric, G. Cartina, G. Grigoras, "Clustering Techniques in Load Profile Analysis for Distribution Stations", Adv Electr Comput Eng, vol. 9, no. 1, pp. 63-66, 2009.

4. D. Q. Hung, N. Mithulananthan, "Loss reduction and loadability enhancement with DG: A dual-index analytical approach", Appl Energ, vol. 115, pp. 233-241, 2014.

5. H. Saadat, "Power Systems Analysis", PSA Publishing, USA, 2010.

6. C. F. Chang, "Reconfiguration and Capacitor Placement for Loss Reduction of Distribution Systems by Ant Colony Search Algorithm", IEEE Trans Power Syst, vol. 23, no. 4, pp. 1747-1755, 2008.

7. O. Elgerd, "Electric Energy Systems Theory: An Introduction", New York: McGraw-Hill, 1971.

8. L. Wang, C. Singh, “Environmental/economic power dispatch using a fuzzified multi-objective particle swarm optimization algorithm", Elec Power Syst Res, vol. 77, no. 12, pp. 1654-1664, 2007.

9. D. Q. Hung, N. Mithulananthan, R. C. Bansal, "Analytical strategies for renewable distributed generation integration considering energy loss minimization", Appl Energ, vol. 105, pp. 75-85, 2013.

10. D. Q. Hung, N. Mithulananthan, R. Bansal, "Analytical Expressions for DG Allocation in Primary Distribution Networks", IEEE Trans Energy Convers, vol. 25, no. 3, pp. 814-820, 2010.

11. D. Q. Hung, N. Mithulananthan, "Multiple Distributed Generator Placement in Primary Distribution Networks for Loss Reduction", IEEE Trans Ind Electron, vol. 60, no. 4, pp. 1700-1708, 2013.

12. M. Shahzad, I. Ahmad, W. Gawlik, P. Palensky, “Load Concentration Factor Based Analytical Method for Optimal Placement of Multiple Distribution Generators for Loss Minimization and Voltage Profile Improvement", Energies, vol. 9, no. 4, p. 287, 2016.

13. N. A. Iqteit, A. B. Arsoy, B. Çakir, "A Simple Method to Estimate Power Losses in Distribution Networks", $10^{\text {th }}$ International Conference on Electrical and Electronics Engineering (ELECO), 2017, pp 135140.

14. N. Mohandas, R. Balamurugan, L. Lakshminarasimman, "Optimal location and sizing of real power DG units to improve the voltage stability in the distribution system using $A B C$ algorithm united with chaos", Int J Elec Power Energ Syst, vol. 66, pp. 41-52, 2015. 


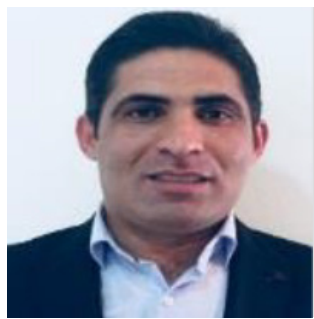

Nassim Ahmad IQTEIT received his B.Sc. in Industrial Automation Engineering from Palestine Polytechnic University (PPU), Palestine, in 2006 and his M.S. degree in Electric Power Engineering from Yarmouk University, Jordan, in 2011. Currently, he is a PhD candidate in Electrical Power Engineering in Kocaeli University, Kocaeli, Turkey. He worked in the Electrical Engineering Department at PPU. His research interests include SEIG modeling, applications of DGs, and distribution system modeling.

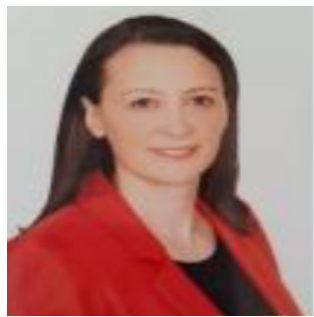

Ayşen BASA ARSOY received her B.Sc. degree from Istanbul Technical University, Turkey, in 1992, an M.S degree from the University of Missouri-Rolla in 1996, and a PhD from Virginia Polytechnic Institute and State University in 2000, all in electrical Engineering. She is currently an Associate Professor at Kocaeli University in Turkey. Her research interests include power system modeling and analysis, power system protection, distributed generation, energy storage, and power electronics applications in Power Systems.

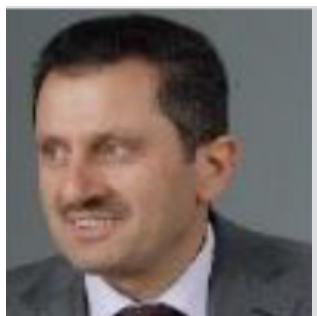

Bekir Çakır received his B.Sc. and M.Sc. degrees from Berlin Technical University, Germany, in 1986 and 1990, respectively, and his PhDs from Yıldız Technical University as well as Kocaeli University, Turkey 1995. In 1991, he joined Kocaeli University's Engineering Faculty as a Research Assistant. He was first Assistant Professor, then Associate Professor at Kocaeli University, 1995 and 2002, respectively. Since 2006, he has been a Professor in the Engineering Faculty, Kocaeli University. His research interests are embedded system design, power electronic driver circuits, renewable energy production systems, and smart grids. 